\title{
Applicability of tetracycline and prednisolone elixir in management of multiple recurrent aphthous ulcers: An original study
}

\author{
Nusrat Nazir ${ }^{1, *}$, Altaf Hussain Chalkoo² \\ ${ }^{1}$ Senior Resident, ${ }^{2}$ Professor \& Head, Dept. of Oral Medicine and Radiology, Govt. Dental College, Srinagar, Jammu \& Kashmir,
} India

*Corresponding Author:

Email: drnusratnaziromar@gmail.com

\begin{abstract}
Introduction: Present study was undertaken to evaluate application of swish and spit [elixir] technique of drug delivery for management of patients with multiple aphthous ulcers especially in large posterior lesions where topical appilication is difficult and cumbersome.

Aim: The aim of present study was to check efficacy of tetracycline and prednisolone elixir in treatment of recurrent aphthous ulcers and check which drug among the two is better for prevention of recurrence of the lesions.

Materials and Methods: A total of fifty patients were selected with recurrent aphthous stomatitis and divided in two groups Group A and Group B. Group A patients were asked to use tetracycline elixir and Group B patients used prednisolone elixir thrice a day for 15 days. Pain Severity, ulcer count and size of the ulcers were determined.

Results: There was no statistically significant difference in study parameters Viz; pain Severity, ulcer count and size of the lesions in both group. However rate of recurrence of lesions was much low in prednisolone group as compare to tetracycline group.

Conclusion: At the end of study it was concluded that both tetracycline and prednisolone elixir can be used for treatment of recurrent aphthous stomatitis. However prednisolone was better in terms of recurrence of lesion with less percentage of recurrent lesions.
\end{abstract}

Keywords: Tetracyline, prednisolone, swish and spit, elixir, multiple recurrent aphthous ulcers.

\section{Introduction}

Among the various oral ulcerative conditions RAS is a very common oral pathosis. ${ }^{1}$ Clinically it presents as solitary or multiple ulcers which are usually shallow oval with erythematous halo and covered by greyish white psuedomembrane. ${ }^{2,3}$ RAS is divided into three types Major, Minor, Herpetiform which can present as simple or complex aphthosis. In complex form of disease severity of pain, ulcer size, and duration is higher. ${ }^{1}$ RAS mainly occur in early childhood and do not disappear later on. RAS mainly occur secondary to some underlying systemic conditions like inflamatory bowel disease, Behcet's disease, Celiac disease and HIV. RAS is often accompanied by fever, malaise and burning sensation at the site of ulcer site prior to ulcer formation..$^{1-4}$ Although the exact etiology of this vague condition is not known TNF-alpha and T- cell mediated immune response has a key role in the pathogenesis of RAS. ${ }^{2,3,5,6}$ Because of unknown etiology and atypical presentations treatment is mainly symptomatic. Lots of drugs have been used in past for the treatment of RAS (eg, minocycline, tetracycline, cephalexin), anti-acidic agents (e.g, sucralfate), and antineoplastics (e.g, thalidomide). ${ }^{7-9}$ In particular, anti-inflammatory and immunomodulatory agents such as clofazimine, thalidomide, pentoxifylline, colchicine, levamisole, azathioprine, amlexanox, and steroids have been administered with varying clinical benefits. ${ }^{10-20}$ Recently prime focus of the treatment of RAS are local and topical application of drugs as there is lower risk of systemic absorption, hence these come under of first line treatment plan, however chances of secondary infections are much higher in case of large posterior ulcers and multiple ulcers where normal salivary flow and tongue movements can displace and rinse the medicament.so the present study was undertaken to check the efficacy of tetracycline and prednisolone elixir for treatment of recurrent aphthous stomatitis.

\section{Materials and Methods}

This study was performed in our department of oral medicine and radiology, government dental college, Srinagar. Fifty patients with RAS were selected. Informed consent was taken from all the patients.

\section{The inclusion criteria consisted of:}

1. Age of patient should be above 18 years.

2. There should be a positive past history of recurrent aphthous ulcers.

3. The duration of ulcer should be less than 36 hours.

The exclusion criteria consisted of:

1. Pregnancy and breast-feeding.

2. Allergy to Tetracycline or prednisolone.

3. Herpetiform aphthous ulcers.

4. All patient with any systemic disease causing oral ulcerations like ulcerative colitis, Cohns disease, Bechets disease, Reiter syndrome, hematological diseases, nutritional deficiencies and allergic conditions.

5. Treatment of the aphthous ulcers with any kind of topical medication in the past 15 days.

6. History of hypertension and diabetes.

7. History of smoking. 
In order to rule out other ulcers from aphthous ulcers, laboratory tests including complete blood cell count, serum iron, serum ferritin and total iron binding capacity were performed for all subjects. Simple random sampling method was used for patient selection, the patients were randomly divided into two groups Group A and Group B consisting of 25 patients each. The two groups were matched according to gender, age, aphthous ulcer count, the level of perceived pain and ulcer duration, size, and location. Two investigators examined the patient after collection of the demographic data. All the findings regarding number, location, size and duration of ulcers as well as pain score using VAS, recurrence time, past and present medical history was also noted. Size of ulcer was measured using $\mathrm{mm}$ caliper. Group A patients used tetracycline oral rinse which was prepared by dissolving tetracycline powder contained within $500 \mathrm{mg}$ capsule of tetracycline in $50 \mathrm{ml}$ of water. Whereas Group B was asked to dissolve $5 \mathrm{mg}$ prednisolone tablet in $50 \mathrm{ml}$ of water. All the patients in both groups were instructed to swish and spit the rinse 3 times in a day for 15 days. All patients were advised not to take food for 30 minutes after using the respective elixir. Two weeks is considered the mean healing time of recurrent aphthous ulcers ${ }^{21}$ therefore the patients were examined 4 times in 15 days on the fifth, eighth, tenth and fifteenth day. Patients were recalled after 1 month and 2 month time interval to check number of episodes of recurrence. In every visit all study parameters were again assessed. In addition any change in pain severity, formation of new ulcers and possible side effects of the drug were evaluated.

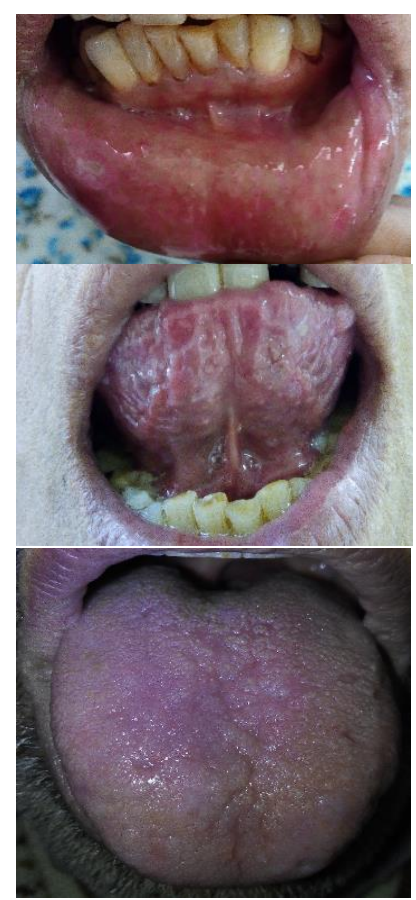

Fig. 1: Clinical photographs of the patients before and after treatment using tetracycline and prednisolone elixir

\section{Results}

In the present study, $38.2 \%$ of the patients were men and $61.8 \%$ were women. Fisher's Exact test was used for gender matching of two groups $(\mathrm{P}>0.05)$. All the patients were in the age group of 18-45 years and matching was confirmed by $\chi 2$ test. Most of the patients had five ulcers (33.3\%), but there was no significant difference in the total number of ulcers between the study groups. In the current study, the severity of the pain, the number of ulcers, and size of the lesions were determined. Severity of pain was measured by VAS criteria. At the initial appointment and also in the next sessions, the mean score of pain severity [Graph 1], mean number of ulcers, mean lesion size in $\mathrm{mm}$ [Graph 2] no statistically significant differences were seen between the two groups
$(\mathrm{P}=1.00)$.However recurrence rate was much lower in prednisolone group as compare to tetracycline group.

\section{Graph 1: Pain score on subsequent visits}

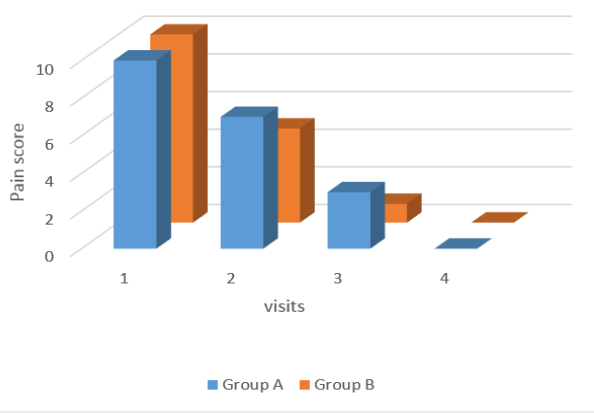




\section{Graph 2: Size of ulcer on subsequent visits}

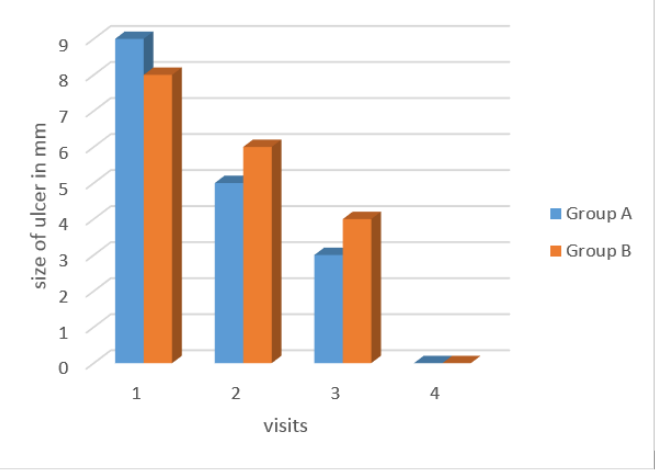

Recurrence was calculated between two groups on 30th and 60th day, Group A showed 29\% recurrence in 30th day and $31 \%$ recurrence in 60th day and Group B showed $16 \%$ recurrence in 30 th day and $18 \%$ recurrence in 60th day.

\section{Discussion}

Recurrent aphthous ulceration is quite common condition affecting the oral cavity, yet it is an important condition since it can be distressing and cause suffering and pain. In addition, it interferes with normal life activities by affecting eating and swallowing of sufferers. The etiology of RAS is unknown, although several factors are suspected including genetics, stress, nutritional deficiencies, diet, hormonal changes, and immunological disorders. ${ }^{22,23}$ Due to the indeterminate etiology of the disease, it is difficult to find a definitive cure and current treatments are aimed towards ameliorating the symptoms. The cause of recurrent aphthous stomatitis is multifactorial and difficult to understood, and treatments are largely empirical and multifaceted. Use of topical tetracycline as an oral rinse reduces the pain associated with the condition. ${ }^{24,25}$ As increased MMP concentrations are detected by the immunostaining of recurrent lesions ${ }^{26}$ it may be suggested that inhibition of MMP relieves the condition. Alternatively, it may be that the prevention of secondary bacterial infection is involved. Tetracycline elixir acts by its antibiotic action. By its ability to block collagenase activity it helps to reduce ulcer size, duration, and pain. ${ }^{27}$ Based on such a strong evidence we also used tetracycline elixir in our study which was prepared by dissolving the powder contained in 500mg capsules of tetracycline in $50 \mathrm{ml}$ of water thrice daily. Results of present study showed that there was reduction in number, size and pain associated with ulcers which was in accordance with Brocklehurst P, Tickle M, Glenny $\mathrm{AM}$, et al. ${ }^{28}$ Same results were seen when prednisolone $5 \mathrm{mg}$ tablets were dissolved in $50 \mathrm{ml}$ of water and used as elixir. A well accepted cause of RAS in genetically susceptible individuals is immune deregulation and that too dysregulation in cell T-cell mediated immune response is mainly behind the pathogenesis of RAS. Corticosteroids may limit the inflammatory process associated with the formation of aphthae. Those medications can act on the lymphocytes and alter the response of effector cells to precipitants of immunopathogenesis (e.g., trauma and food allergies). This may be the reason for low recurrence rate of ulcers in Group B as compare to Group A.

\section{Conclusion}

The findings of present study indicate that both prednisolone and tetracycline elixir can be used in situation of multiple RAS where topical application of drug is difficult and cumbersome. Moreover there is considerable decrease in size and duration of ulcer as well as pain. Both the drugs are equally efficacious in terms of healing time, however recurrence is much less in case of prednisolone as compare to tetracycline. So the concept of prednisolone swish and spit technique has tremendous scope for future research in management of this intractable condition.

\section{References}

1. Baccaglini L, Lalla RV, Bruce AJ, et al. Urban legends: recurrent aphthous stomatitis. Oral Dis 2011;17(8):75570.

2. Scully C, Shotts R. ABC of oral health. Mouth ulcers and other causes of orofacial soreness and pain. $B M J$ 2000;321(7254):162-5.

3. Gonsalves WC, Chi AC, Neville BW. Common oral lesions: Part I.Superficial mucosal lesions. Am Fam Physician. 2007;75(4):501-7.

4. Scully C, Hodgson T. Recurrent oral ulceration: aphthous-like ulcers in periodic syndromes. Oral Surg Oral Med Oral Pathol Oral Radiol Endod 2008;106(6):845-852.

5. Brice SL. Clinical evaluation of the use of low-intensity ultrasound in the treatment of recurrent aphthous stomatitis. Oral Surg Oral Med Oral Pathol Oral Radiol Endod 1997;83(1):14-20.

6. Eversole LR. Immunopathogenesis of oral lichen planus and recurrent aphthous stomatitis. Semin Cutan Med Surg 1997;16(4):284-94.

7. Gorsky M, Epstein J, Rabenstein S, Elishoov H, Yarom $\mathrm{N}$. Topical minocycline and tetracycline rinses in treatment of recurrent aphthous stomatitis: a randomized cross-over study. Dermatol Online J. 2007;13(2):1.

8. Gorsky M, Epstein J, Raviv A, Yaniv R, Truelove E. Topical minocycline for managing symptoms of recurrent aphthous stomatitis. Spec Care Dentist 2008;28(1):27-31.

9. Chiu HY, Tsai TF. Topical use of systemic drugs in dermatology: a comprehensive review. J Am Acad Dermatol 2011;65(5):1048. e1-e22.

10. de Abreu MA, Hirata CH, Pimentel DR, Weckx LL. Treatment of recurrent aphthous stomatitis with clofazimine. Oral Surg Oral Med Oral Pathol Oral Radiol Endod 2009;108(5):714-21.

11. Escudier M, Nunes C, Sanderson JD. Disorders of the mouth. Med. 2011;39(3):127-31.

12. Thornhill MH, Baccaglini L, Theaker E, Pemberton MN. A randomized, double-blind, placebo-controlled trial of pentoxifylline for the treatment of recurrent aphthous stomatitis. Arch Dermatol 2007;143(4):463-70.

13. Davatchi F, Shahram F, Chams-Davatchi C, et al. How to deal with Behcet's disease in daily practice. Int J Rheum Dis 2010;13(2):105-16. 
14. Pakfetrat A, Mansourian A, Momen-Heravi F, et al. Comparison of colchicine versus prednisolone in recurrent aphthous stomatitis: A double-blind randomized clinical trial. Clin Invest Med. 2010;33(3): E189-E195.

15. Picciani BL, Silva-Junior GO, Barbirato DS, Ramos RT, Cantisano MH. Regression of major recurrent aphthous ulcerations using a combination of intralesional corticosteroids and levamisole: a case report. Clinics (Sao Paulo). 2010;65(6):650-52.

16. Liu J, Zeng X, Chen Q, et al. An evaluation on the efficacy and safety of amlexanox oral adhesive tablets in the treatment of recurrent minor aphthous ulceration in a Chinese cohort: a randomized, double-blind, vehiclecontrolled, unparallel multicenter clinical trial. Oral Surg Oral Med Oral Pathol Oral Radiol Endod. 2006;102(4):475-81.

17. Murray B, Biagioni PA, Lamey PJ. The efficacy of amlexanox OraDisc on the prevention of recurrent minor aphthous ulceration. J Oral Pathol Med. 2006;35(2):11722.

18. Lin P, Liang G. Behçet disease: recommendation for clinical management of mucocutaneous lesions. J Clin Rheumatol. 2006;12(6):282-6.

19. Femiano F, Buonaiuto C, Gombos F, Lanza A, Cirillo N. Pilot study on recurrent aphthous stomatitis (RAS): a randomized placebo-controlled trial for the comparative therapeutic effects of systemic prednisone and systemic montelukast in subjects unresponsive to topical therapy. Oral Surg Oral Med Oral Pathol Oral Radiol Endod. 2010;109(3):402-7.

20. Elad S, Epstein JB, von Bültzingslöwen I, Drucker S, Tzach R, Yarom N. Topical immunomodulators for management of oral mucosal conditions, a systematic review; Part II: miscellaneous agents. Expert Opin Emerg Drugs. 2011;16(1):183-202.

21. Neville B, Damm D, Allen G, Bouquot J. Oral \& Maxillofacial Pathology. 2nd ed. Philadelphia: WB. Saunders; 2002. p.243,285-91.

22. Scully C. The diagnosis and management of recurrent aphthous stomatitis: a consensus approach. $J$ Am Dent Ass 2003; 134:200-7.

23. Scully $\mathrm{C}$ and Felix DH. Oral medicine: update for the dental practioner. Aphthous and other common ulcers. $\mathrm{Br}$ Dent J 2005; 199(5):259-64.

24. Henricsson V, Axéll T. Treatment of recurrent aphthous ulcers withaureomycin mouth rinse or zendium dentifrice. Acta Odontol Scand1985;43:47-52.

25. Ylikontiola L, Sorsa T, Häyrinen-Immonen R, Salo T.Doxymycine-cyanoacrylate treatment of recurrent aphthous ulcers.Oral Surg Oral Med Oral Pathol Oral Radiol Endod 1997;83:329-33.

26. Häyrinen-Immonen R, Sorsa T, Nordström D, Malmström M, KonttinenYT. Collagenase and stromelysin in recurrent aphthous ulcers (RAU). IntJ Oral Maxillofac Surg 1993;22:46-9.

27. Ship JA, Chavez EM, Doerr PA, Henson BS, Sarmadi M. Recurrent aphthous stomatitis. Quintessence Int 2000;31(2):95-112.

28. Brocklehurst P, Tickle M, Glenny AM, et al. Systemic interventions for recurrent aphthous stomatitis. 